\title{
Necessidades de familiares de pacientes internados em unidade de cuidados intensivos
}

\author{
Needs of family members of patients admitted to an intensive care unit \\ Necesidades de los familiares de pacientes internados en unidad de cuidados intensivos
}

\section{Marina Rumiko Maruiti', Luiza Elaine Galdeano²}

\begin{abstract}
RESUMO
Objetivo: Identificar as necessidades de familiares de pacientes internados em uma unidade de cuidados intensivos. Métodos: Fizerem parte da amostra 39 familiares de pacientes em estado crítico de saúde. Para a coleta de dados foi utilizada uma adaptação do Critical Care Family Need Inventory . Resultados: a necessidade de conhecimento/informação identificada com maior freqüência foi saber fatos concretos sobre o progresso do paciente (84,6\%); a necessidade de conforto identificada com maior freqüência foi ter um toalete e um telefone próximo à sala de espera (56,4\%); a necessidade de segurança identificada com maior freqüência foi ter a certeza que o paciente está recebendo o melhor tratamento (89,7\%); e a necessidade de acesso ao paciente e aos profissionais da unidade com maior freqüência foi conversar com o médico todos os dias (79,5\%). Conclusão: Os profissionais de enfermagem devem se preocupar em atender não apenas as necessidades dos pacientes, mas também de seus familiares.

Descritores: Relações profissional-família; Determinação de necessidades de cuidados de saúde; Cuidados de Enfermagem; Unidades de terapia intensiva
\end{abstract}

\begin{abstract}
Objective: To identify the needs of family members of patient admitted to an intensive care unit. Methods: A sample of 39 family members participated in the study. Data were collected with a modified form of the Critical Care Family Need Inventory. Results: The most frequent needs reported by family members were: to be certain that their loved ones were receiving the best treatment (89.7\%), to receive concrete information about the progress of their relatives (84.6\%), to speak with the physician everyday (79.5\%), and to have restrooms and telephones close to the waiting room $(56.4 \%)$. Conclusion: Beyond providing quality care to critical care patients, nursing staff must be sensitive to the needs of patients' family members as well.
\end{abstract}

Keywords: Professional-family relations; Needs assessment; Nursing care; Intensive care units

\section{RESUMEN}

Objetivo: Identificar las necesidades de los familiares de pacientes internados en una unidad de cuidados intensivos. Métodos: Formaron parte de la muestra 39 familiares de pacientes en estado crítico de salud. Para la recolección de datos fue utilizada una adaptación del Critical Care Family Need Inventory. Resultados: La necesidad de conocimiento/información identificada con mayor frecuencia fue saber hechos concretos sobre el progreso de paciente (84,6\%); la necesidad de comodidad identificada con mayor frecuencia fue tener un baño y teléfono próximo a la sala de espera (56,4\%); la necesidad de seguridad registrada con mayor frecuencia fue tener la certeza de que lo patiente está recibiendo el mejor tratamiento $(89,7 \%$ ) y la necesidad de acceso al paciente y a los profesionales de la unidad detectada con mayor frecuencia fue hablar con el médico todos los días (79,5\%). Conclusíon: La enfermería no debe preocuparse solamente con las necesidades de los pacientes, sino también con las de sus familiares.

Descriptores: Relaciones profesional-familia; Evaluación de necesidades; Atención de enfermería; Unidades de terapia intensiva

\footnotetext{
${ }^{1}$ Enfermeira da Empresa AxisMed - Gestão Preventiva de Saúde de São Paulo.São Paulo (SP), Brasil.

${ }^{2}$ Professor Assistente da Faculdade de Enfermagem do Hospital Israelita Albert Einstein - HLAE - São Paulo (SP), Brasil.
} 


\section{INTRODUÇÃO}

A Unidade de Terapia Intensiva (UTI) é uma unidade fechada, isto é, uma unidade que facilita a coordenação das atividades dos profissionais que ali trabalham e que restringe o acesso a outras pessoas. A planta física e os equipamentos característicos despertam curiosidade a todos que adentram nesse território. A estrutura física da UTI, associada às condições dos pacientes, normalmente críticas, e a intensa atividade da equipe de saúde, fazem com que muitas pessoas considerem essa unidade um ambiente hostil $^{(1)}$. Sem cadeiras para familiares, logo, sem um convite para uma visita prolongada ${ }^{(2)}$, a entrada de familiares, é permitida durante curto período de tempo e em horários pré-estabelecidos. Além disso, não se observa a preocupação com o preparo prévio do familiar para o contato com o paciente e com o "novo ambiente"(1).

Em um estudo sobre o significado cultural do cuidado em UTI, a pesquisadora observou que o relacionamento da equipe de enfermagem com os familiares foi formal, burocrático e, sobretudo, despersonalizado; observou que o diálogo com a família foi superficial, que o enfermeiro estava ausente a maior parte do tempo, e que os outros funcionários da unidade evitavam o contato com os familiares ${ }^{(3)}$.

A humanização do cuidado de enfermagem na UTI vai além de permitir ou não a visita do familiar, inclui também o estabelecimento de uma relação de confiança e de ajuda, na qual a equipe de enfermagem tem a função de identificar as reais necessidades dos familiares. Quanto mais cedo a interação (enfermeiro/familiares) ocorrer melhor será para a família e, conseqüentemente, para o paciente ${ }^{(4)}$.

Em um outro estudo sobre a comunicação dos profissionais de saúde com os familiares em UTIs concluiu-se que entre os fatores que dificultam esse processo está o desconhecimento por parte dos profissionais do modo de ser e de perceber da família ${ }^{(2)}$.

Em geral, observa-se que o foco da assistência de enfermagem é o atendimento às necessidades do paciente. No entanto, o paciente não é o único a sofrer com a doença e com a hospitalização, os familiares e outras pessoas envolvidas diretamente com o paciente, compartilham a angústia, o medo e o sofrimento desse momento. Sendo assim, é importante que o profissional de saúde dispense atenção a aos familiares, com o objetivo de facilitar o enfrentamento dessa nova experiência.

A assistência de enfermagem deve atender às necessidades dos pacientes e familiares, ajudando-os a compreender, a aceitar e a enfrentar a doença, o tratamento e as conseqüências que essa nova situação impõe à vida familiar.

\section{OBJETIVO}

Identificar as necessidades de familiares de pacientes internados em uma unidade de cuidados intensivos.

\section{MÉTODOS}

Trata-se de um estudo descritivo, exploratório, de abordagem quantitativa, desenvolvido na sala de espera da UTI de um hospital geral, particular, de grande porte, situado na zona Sul do Município de São Paulo.

A amostra foi obtida por conveniência, sendo constituída por 39 familiares de pacientes que se encontravam internados na UTI. Os critérios para inclusão no estudo foram: ser adulto, possuir familiar internado na UTI, independente do grau de parentesco e concordar voluntariamente em participar da pesquisa, mediante a assinatura do Termo de Consentimento Livre e Esclarecido.

$O$ instrumento de coleta de dados elaborado para esse estudo consta de duas partes (Anexo A). A primeira parte consiste de Dados de Identificação, para caracterização da amostra. A segunda parte do instrumento foi elaborada a partir de uma adaptação do Critical Care Family Need Inventory (CCFNI) ${ }^{(5)}$, um inventário constituído de 45 itens para identificar as necessidades de familiares de pacientes em estado crítico de saúde.

O CCFNI foi publicado em $1979^{(5)}$ e validado por vários pesquisadores de outros países ${ }^{(6-8)}$. Em um estudo nacional ${ }^{(9)}$ foi realizada a validação da escala de razão de um conjunto de necessidades do CCFNI.

Para este estudo, os 45 itens do CCFNI foram organizados em quatro categorias de necessidades, a saber: necessidade de conhecimento/informação, na qual foram agrupados os itens relacionados à necessidade dos familiares de receber informações das mais variadas, ou de obter conhecimento relacionado ao estado de saúde ou aos procedimentos envolvidos na terapêutica do familiar doente; necessidade de conforto, relacionado ao planejamento físico da unidade, mobília nela contida e necessidade dos familiares em sentirem-se confortáveis na sala de espera ou na própria UTI durante a visita; necessidade de segurança emocional, na qual foram agrupados os itens relacionados à necessidade dos familiares de sentirem-se seguros, menos ansiosos e/ou temerosos quanto ao estado de saúde e ao prognóstico de seu familiar; e necessidade de acesso ao paciente e aos profissionais da unidade na qual foram agrupados os itens relacionados à necessidade dos familiares em ter mais acesso aos profissionais da instituição (médicos, enfermeiros, fisioterapeutas, nutricionistas, psicólogos e outros funcionários não relacionados à área médica).

A coleta de dados foi realizada em setembro de 2005, após a aprovação pelo Comitê de Ética em Pesquisa do Hospital Israelita Albert Einstein e se procedeu da seguinte maneira: a pesquisadora abordou familiares na sala de 
espera, após já terem realizado a visita, e indagou de cada um a vontade da participação no estudo. Havendo uma confirmação, mediante a assinatura do Termo de Consentimento Livre e Esclarecido, foi entregue o instrumento de coleta de dados (Anexo A), sendo fornecidas as instruções necessárias para o preenchimento do mesmo.

Os dados coletados foram organizados e sintetizados a partir da estatística descritiva e apresentados em números absolutos e percentuais.

\section{RESULTADOS}

Dos 39 familiares que participaram do estudo, 28 $(72 \%)$ eram do sexo feminino e 11 (28\%) do sexo masculino. A média de idade dos participantes foi 37 anos.

Tabela 1 - Necessidade de conhecimento/ informação de familiares de pacientes internados em UTI, de um hospital geral, particular. São Paulo, SP, set 2005

\begin{tabular}{|c|c|c|}
\hline Necessidade de conhecimento / informação & $\mathbf{N}^{\mathbf{o}}$ & $\%$ \\
\hline $\begin{array}{l}\text { Saber fatos concretos sobre o progresso do } \\
\text { paciente }\end{array}$ & 33 & 84,6 \\
\hline $\begin{array}{l}\text { Saber exatamente o que está sendo feito para o } \\
\text { paciente }\end{array}$ & 32 & 82,1 \\
\hline Ter os questionamentos respondidos claramente & 29 & 74,4 \\
\hline $\begin{array}{l}\text { Receber informação sobre o estado de saúde do } \\
\text { paciente pelo menos uma vez por dia }\end{array}$ & 29 & 74,4 \\
\hline $\begin{array}{l}\text { Conhecer os profissionais que estão cuidando do } \\
\text { paciente }\end{array}$ & 25 & 64,1 \\
\hline $\begin{array}{l}\text { Saber o porquê determinado procedimento foi } \\
\text { realizado }\end{array}$ & 23 & 59,0 \\
\hline $\begin{array}{l}\text { Ser informado (a) em casa sobre mudanças nas } \\
\text { condições do paciente }\end{array}$ & 22 & 56,4 \\
\hline $\begin{array}{l}\text { Saber quais são as medicações que o paciente está } \\
\text { fazendo uso }\end{array}$ & 21 & 53,8 \\
\hline Conhecer a expectativa de alta & 20 & 51,3 \\
\hline Prover de explicações compreensíveis & 19 & 48,7 \\
\hline $\begin{array}{l}\text { Receber orientações como o quê fazer ao lado da } \\
\text { cama do paciente }\end{array}$ & 18 & 46,2 \\
\hline $\begin{array}{l}\text { Saber qual membro da equipe pode dar cada tipo } \\
\text { de informação }\end{array}$ & 18 & 46,2 \\
\hline $\begin{array}{l}\text { Ser informado (a) sobre os planos de transferência } \\
\text { enquanto estes estão sendo realizados }\end{array}$ & 17 & 43,6 \\
\hline $\begin{array}{l}\text { Ter explicações sobre o ambiente da UTI antes de } \\
\text { adentrá-la pela primeira vez }\end{array}$ & 15 & 38,5 \\
\hline $\begin{array}{l}\text { Ser informado (a) sobre outras pessoas que } \\
\text { poderiam ajudar com os problemas }\end{array}$ & 13 & 33,3 \\
\hline $\begin{array}{l}\text { Ser avisado (a) sobre os serviços religiosos } \\
\text { existentes na instituição }\end{array}$ & 11 & 28,2 \\
\hline $\begin{array}{l}\text { Ser informado (a) sobre alguém que possa ajudar } \\
\text { nos problemas familiares }\end{array}$ & 8 & 20,5 \\
\hline
\end{tabular}

A partir dos dados apresentados na Tabela 1 pode-se estimar a necessidade que os membros da família, de um paciente em estado crítico de saúde, possui em ter informações precisas, isto é, de saber o que está de fato acontecendo com o seu familiar hospitalizado.

Tabela 2 - Necessidade de conforto de familiares de pacientes internados em UTI, de um hospital geral, particular. São Paulo, SP, set 2005

\begin{tabular}{lcc}
\hline Necessidade de conforto & $\mathbf{N}^{\mathbf{o}}$ & $\%$ \\
\hline Ter um toalete próximo à sala de espera & 22 & 56,4 \\
Ter um telefone próximo à sala de espera & 22 & 56,4 \\
Ter uma mobília confortável na sala de espera & 21 & 53,8 \\
Ter a sala de espera próxima ao paciente & 20 & 51,3 \\
Ter disponível no hospital uma boa comida & 18 & 46,2 \\
Ter um lugar no hospital onde possa ficar & 14 & 35,9 \\
sozinho (a) & & \\
\hline
\end{tabular}

Estar em um lugar agradável, que ofereça conforto enquanto aguarda para receber notícias de seu familiar internado, constitui, segundo os dados apresentados na Tabela 2, um fator importante para a maioria dos participantes desse estudo.

Tabela 3 - Necessidade de segurança emocional de familiares de pacientes internados em UTI, de um hospital geral, particular. São Paulo, SP, set 2005

\begin{tabular}{|c|c|c|}
\hline Necessidade de segurança emocional & $\mathbf{N}^{\mathbf{0}}$ & $\%$ \\
\hline $\begin{array}{l}\text { Ter a certeza que o paciente está recebendo o } \\
\text { melhor tratamento }\end{array}$ & 35 & 89,7 \\
\hline Sentir-se acolhido pela equipe hospitalar & 31 & 79,5 \\
\hline Sentir que há esperança & 27 & 69,2 \\
\hline Ter amigos próximos para suporte & 18 & 46,2 \\
\hline $\begin{array}{l}\text { Conversar sobre sentimentos em relação ao que } \\
\text { vem acontecendo }\end{array}$ & 16 & 41,0 \\
\hline Sentir-se à vontade para chorar & 15 & 38,5 \\
\hline $\begin{array}{l}\text { Conversar sobre as possibilidades de } \\
\text { falecimento do paciente }\end{array}$ & 12 & 30,8 \\
\hline $\begin{array}{l}\text { Ter a liberdade de ficar sozinho (a) a qualquer } \\
\text { hora }\end{array}$ & 12 & 30,8 \\
\hline $\begin{array}{l}\text { Ter outra pessoa próxima quando estiver } \\
\text { visitando o paciente na UTI }\end{array}$ & 11 & 28,2 \\
\hline $\begin{array}{l}\text { Ser assegurado (a) em relação à segurança } \\
\text { enquanto estiver no hospital }\end{array}$ & 10 & 25,6 \\
\hline Ter alguém encarregado sobre sua saúde & 9 & 23,1 \\
\hline $\begin{array}{l}\text { Receber a visita de um representante religioso } \\
\text { (padre, pastor, rabino...) }\end{array}$ & 8 & 20,5 \\
\hline
\end{tabular}

A família se preocupa com o paciente e vivencia o medo e a insegurança, muitas vezes resultado da incerteza em relação à conduta e ao tratamento. A dúvida e o questionamento quanto a melhor opção de hospital, de profissional e, em algumas situações, de terapêutica, é quase inevitável, causando o sofrimento da família.

É importante ressaltar que nenhum dos 39 familiares acrescentou outras necessidades, além daquelas já contidas no instrumento.

\section{DISCUSSÃO}

De acordo com a Tabela 1, a necessidade de conhecimento/informação relatada pelo maior número de familiares foi saber fatos concretos sobre o progresso do meu familiar, relatada por $33(84,6 \%)$ indivíduos, seguido por saber exatamente o que está sendo feito para 
o paciente, relatado por 32 (82,1\%). Esses resultados são similares aos resultados encontrados em um estudo, no qual foram levantadas as necessidades de pacientes internados em uma UTI ortopédica ${ }^{(10)}$.

Tabela 4 - Necessidade de maior acesso ao paciente e aos profissionais da unidade de pacientes internados em UTI, de um hospital geral, particular. São Paulo, SP, set 2005

\begin{tabular}{|c|c|c|}
\hline $\begin{array}{l}\text { Necessidade de maior acesso ao paciente e } \\
\text { aos profissionais da unidade }\end{array}$ & $\mathbf{N}^{\mathbf{0}}$ & $\%$ \\
\hline Conversar com o médico todos os dias & 31 & 79,5 \\
\hline Ver o paciente freqüentemente & 30 & 76,9 \\
\hline $\begin{array}{l}\text { Ter o dia e horário de visita flexível, podendo ser } \\
\text { modificado em situações especiais }\end{array}$ & 28 & 71,8 \\
\hline $\begin{array}{l}\text { Ter uma pessoa específica no hospital para entrar } \\
\text { em contato e dar notícias do paciente, quando não } \\
\text { puder estar presente na visita }\end{array}$ & 25 & 64,1 \\
\hline $\begin{array}{l}\text { Conversar com a enfermeira responsável todos os } \\
\text { dias }\end{array}$ & 22 & 56,4 \\
\hline $\begin{array}{l}\text { Conhecer os cuidados da equipe hospitalar com } \\
\text { relação ao paciente }\end{array}$ & 21 & 53,8 \\
\hline $\begin{array}{l}\text { Ter o horário de início da visita respeitado, isto é, } \\
\text { iniciar a visita pontualmente no horário previsto }\end{array}$ & 18 & 46,2 \\
\hline $\begin{array}{l}\text { Ajudar com relação aos cuidados físicos do } \\
\text { paciente }\end{array}$ & 16 & 41,0 \\
\hline Poder visitar a qualquer momento & 12 & 30,8 \\
\hline $\begin{array}{l}\text { Ter alguém para ajudar com o problema } \\
\text { financeiro }\end{array}$ & 7 & 17,9 \\
\hline
\end{tabular}

Sabe-se que a falta de informação e a incerteza constituem importantes fontes de ansiedade em pacientes e em seus familiares ${ }^{(11)}$. A incerteza normalmente causa apreensão e ansiedade nos familiares, que esperam aflitos o momento da visita para retirarem as suas dúvidas e, de preferência, receberem boas notícias. Nesse caso, entendese por "boas notícias" informações relacionadas à melhora do estado clínico do paciente, ao aumento da probabilidade de cura e à diminuição do risco de morte.

A expectativa dos familiares de receber "boas notícias" é sempre muito grande, no entanto, o profissional dever ter o compromisso ético de fornecer informações verdadeiras, sejam elas boas ou más. Para isso, o profissional deve ter a habilidade e a sensibilidade de perceber a capacidade do paciente de seus familiares de compreender as informações de enfrentar a situação vivenciada, uma vez que comunicar uma verdade de forma inadequada, por um profissional despreparado, pode ser tão prejudicial como ocultá-la ${ }^{(12)}$.

A ação educativa do enfermeiro pode diminuir o desconforto e a ansiedade dos familiares. Essa ação não deve restringir-se a informar o paciente e a sua família quanto a natureza e as causas da doença, bem como os riscos relacionados à terapêutica. O enfermeiro deve estar preparado para estabelecer um relacionamento de empatia e confiança com a família, para comunicar-se de forma adequada, a fim de incentivar e motivar os familiares a retirarem todas as suas dúvidas, de forma a satisfazer a necessidade de informação e, desse modo, diminuir a angústia e o sofrimento de todos os envolvidos.

Quanto à necessidade de conforto, observa-se que das seis necessidades, apresentadas na Tabela 2, quatro foram citadas por mais de $50 \%$ dos familiares. Esses resultados demonstram a importância que os familiares dão ao espaço físico e à mobília da sala de espera de uma UTI.

É conveniente reiterar que os dados deste estudo foram coletados em um hospital particular, diferenciado, no qual existe uma preocupação com o ambiente e com o conforto dos visitantes. Isso nos faz refletir sobre o grau de importância que outras pessoas (como, por exemplo, aquelas que vivenciam a experiência de ter um familiar internado em uma unidade de cuidados críticos de um hospital sem recursos, onde, geralmente no qual não há essa preocupação com o espaço físico e com o conforto) dão a esse tipo de necessidade.

Ter a certeza que o paciente está recebendo o melhor tratamento foi a necessidade de segurança emocional relatada por maior número de familiares. Esse resultado reflete a necessidade do ser humano de sentir-se seguro e, conforme discutido anteriormente, de certeza de que tudo irá correr bem, uma vez que a dúvida é fonte de sentimentos desagradáveis, como a ansiedade e a angústia.

Das 10 necessidades apresentadas na Tabela 4, relacionadas às necessidades de maior acesso ao paciente e aos profissionais da unidade, seis foram relatadas por mais de 50\% dos familiares, a saber: conversar com o médico todos os dias $(79,5 \%)$; ver o paciente freqüentemente $(76,9 \%)$; ter o dia e o horário de visita flexíveis podendo ser modificados em situações especiais (71,8\%); ter uma pessoa específica no hospital para entrar em contato e dar notícias do paciente, quando não puder estar presente na visita $(64,1 \%)$; conversar com a enfermeira responsável todos os dias $(56,4 \%)$; conhecer os cuidados da equipe hospitalar com relação ao paciente $(53,8 \%)$. Esses resultados demonstram a importância que os familiares dão às informações e ao contato direto com os profissionais que cuidam do seu ente querido. Esses dados também vão ao encontro de outros resultados encontrados na literatura.

Em um estudo, no qual foi realizado um levantamento da percepção do familiar do paciente crítico sobre a comunicação com a equipe de enfermagem, identificouse que as orientações e o contato com o enfermeiro são muito importantes e que, segundo os familiares, esse profissional deveria explicar de forma clara e objetiva o que está acontecendo com o paciente, como ele evoluiu e o que significa estar estável. Nesse mesmo estudo, concluiu-se que os familiares apresentam dificuldades em ter acesso a quem detém as informações, e que o ideal seria existir uma pessoa devidamente treinada, que soubesse de cada caso, para transmitir as informações necessárias ${ }^{(13)}$. 


\section{CONCLUSÃO}

Sendo o paciente o foco do cuidado, as necessidades dos familiares são, muitas vezes, desconhecidas pela equipe de enfermagem. A sensibilidade do enfermeiro em perceber as necessidades da família pode resultar na implementação de novas políticas, como horário de visitas mais flexíveis, maior proximidade da equipe de enfermagem e maior facilidade na obtenção de informações.

Os resultados identificados nesse estudo propiciam a reflexão sobre a relação enfermeiro-familiar e sua repercussão na assistência a pacientes em estado crítico de saúde e permitem concluir que as necessidades relatadas com maior freqüência recaíram nas categorias "conhecimento/informação" e "segurança emocional", representadas por saber fatos concretos sobre o progresso do paciente $(84,6 \%)$ e ter a certeza que o paciente está recebendo o melhor tratamento $(89,7 \%)$ respectivamente. Sendo assim, é fundamental que o enfermeiro se empenhe em fornecer informações fidedignas, claras e objetivas, sobre o estado de saúde dos pacientes internados em UTI e, dessa forma, tentar diminuir os sentimentos de insegurança e angústia da família. O enfermeiro deve atuar, também, no sentido de reforçar os mecanismos de enfrentamento desses familiares, com o objetivo de fornecer apoio emocional e mobilizar sentimentos positivos.

A enfermagem, sendo uma profissão que enfatiza o cuidado personalizado e holístico, deve se preocupar em atender, não apenas as necessidades dos pacientes, mas também de seus familiares.

\section{REFERÊNCIAS}

1. Takahashi EIU Visitas em unidade de terapia intensiva. Rev
Paul Enfermagem. 1986; 6(3): 113-5.

2. Santos KMAB. Percepção dos profissionais de saúde sobre a comunicação com os familiares de pacientes em UTIs e análise proxêmica dessas interações [tese]. São Paulo: Escola de Enfermagem da Universidade de São Paulo; 2004

3. Vila VSC. O significado cultural do cuidado humanizado em unidade de terapia intensiva: muito falado e pouco vivido [tese]. Ribeirão Preto: Escola de Enfermagem de Ribeirão Preto da Universidade de São Paulo; 2001.

4. Pauli MC, Bousso RS. Crenças que permeiam a humanização da assistência em unidade de terapia intensiva pediátrica. Rev Latinoam Enfermagem. 2003; 11(3): 280-6.

5. Molter NC. Needs of relatives of critically ill patients: a descriptive study. Heart Lung. 1979; 8(2): 332-9.

6. Leske JS. Internal psychometric properties of the Critical Care Family Needs Inventory. Heart Lung. 1991; 20(3): 236-44.

7. Bijttebier P, Delva D, Vanoost S, Bobbaers H, Lauwers P, Ventommen H.. Reliability and validity of the Critical Care Family Needs Inventory in a Dutch-speaking Belgian sample. Heart Lung. 2000; 29(4): 278-86.

8. Neabel B, Fothergill-Bourbonnais F, Dunnung J. Family assessment tools: a review of the literature from 1978-1997. Heart Lung. 2000; 29(3): 196-209.

9. Morgon FH, Guirardello EB. Validação da escala de razão das necessidades de familiares em unidade de terapia intensiva. Rev Latinoam Enfermagem. 2004; 12(2): 198-203.

10. Garcia PC, Faro ACM. Necessidades e expectativas de familiares de pacientes em Unidades de Terapia Intensiva de ortopedia. Rev Paul Enfermagem. 2004; 23(1): 57-64.

11. Fitzsimons D, Parahoo K, Stringer M. Waiting for coronary artery bypass surgery: a qualitative analysis. J Adv Nurs. 2000; 32(5):1243-52.

12. Bascuñán R ML. Comunicación de la verdad en medicina: contribuiciones desde una perspectiva psicológica. Rev Med Chile. 2005; 133(6): 693-8.

13. Inaba LC, Silva MJP, Telles SCR. Paciente crítico e comunicação: visão de familiares sobre sua adequação pela equipe de enfermagem. Rev Esc Enfermagem USP. 2005; 39(4): 426-9. 


\section{Anexo A: Instrumento de coleta de dados}

\section{$1^{\mathrm{a}}$ Parte: Dados de identificação}

Iniciais do nome: Idade:

Sexo: ( ) masculino ( )feminino Religião:

Grau de instrução: () Fundamental completo () Médio completo () Superior () Outros

Tipo de parentesco com o paciente: Tempo de internação do paciente:

\section{$2^{\mathrm{a}}$ Parte: Inventário das necessidades de familiares de pacientes em estado crítico de saúde}

Este inventário tem como objetivo identificar quais são as suas necessidades enquanto pessoa que possui um ente querido internado em uma unidade de terapia intensiva.

$\mathrm{O}$ (a) Sr(a) deverá ler o que significa cada necessidade e marcar com um " $\mathrm{X}$ " nas respostas que melhor correspondem as suas necessidades.

Assim como no questionário anterior, não é preciso ficar pensando muito em cada questão, pois, neste questionário, também as respostas espontâneas têm maior valor do que aquelas em que se pensa muito.

$\mathrm{O}$ (a) Sr(a) poderá marcar quantas respostas forem necessárias e ainda acrescentar outras necessidades que não estejam descritas no questionário. Essas outras necessidades poderão ser escritas no espaço intitulado "Outras necessidades".

A) Necessidade de conhecimento/ informação: referem-se às necessidades de receber informações ou de obter conhecimento relacionado ao estado de saúde, ou aos procedimentos envolvidos no tratamento do seu ente querido hospitalizado.

( ) 1. Conhecer expectativa de alta

( ) 2. Ter meus questionamentos respondidos claramente

( ) 3. Ser informado em casa sobre mudanças da condição do meu familiar

( ) 4. Saber quais são as medicações que o meu familiar está fazendo uso

( ) 5. Saber fatos concretos sobre o progresso do meu familiar

( ) 6. Receber informação sobre o estado de saúde do meu familiar pelo menos uma vez por dia

( ) 7. Ser provido de explicações compreensíveis

( ) 8. Saber o porquê determinado procedimento foi realizado

( ) 9. Saber exatamente o que está sendo feito pelo meu familiar

( ) 10. Ser informado sobre outras pessoas que poderiam ajudar com os problemas

( ) 11.Receber orientações como o quê fazer ao lado da cama do meu familiar doente

( ) 12. Saber qual membro da equipe multidisciplinar pode dar determinada informação

( ) 13. Ter explicação sobre o ambiente da UTI antes de adentrá-la pela primeira vez

( ) 14. Conhecer os profissionais que estão cuidando do meu familiar

( ) 15. Ser informado(a) sobre alguém que possa ajudar com problemas familiares

( ) 16. Ser informado(a) sobre planos de transferência enquanto estes estão sendo realizados

( ) 17. Ser avisado(a) sobre os serviços religiosos existentes na instituição

Outras necessidades (nesse espaço, o $\operatorname{Sr}(\mathrm{a})$ poderá acrescentar outros itens que correspondem a outras necessidades suas, relacionadas a conhecimento/ informação, que não constam na listagem apresentada acima): 
B) Necessidade de conforto: referem-se às necessidades para sentir-se confortável na sala de espera ou na própria UTI durante a visita, os itens dessa categoria estão relacionados ao planejamento físico da unidade e a mobília nela contida.

( ) 1 . Ter um toalete próximo à sala de espera

( ) 2. Ter um telefone próximo à sala de espera

( ) 3. Ter a sala de espera próxima do meu familiar

( ) 4. Ter uma mobília confortável na sala de espera

( ) 5. Ter um lugar no hospital onde possa ficar sozinho(a)

( ) 6. Ter disponível no hospital uma boa comida

Outras necessidades (nesse espaço, o $\mathrm{Sr}(\mathrm{a})$ poderá acrescentar outros itens que correspondem a outras necessidades suas, relacionadas ao conforto, que não constam na listagem apresentada acima):

C) Necessidade de segurança emocional: referem-se às necessidades de se sentir seguro(a), de se sentir menos ansioso(a) e menos temeroso(a) quanto ao estado de saúde e ao prognóstico de seu ente querido.

( ) 1. Ter a certeza que o meu familiar está recebendo o melhor tratamento

( ) 2. Sentir que há esperança

( ) 3. Ter amigos próximos para suporte

( ) 4. Sentir-se acolhido pela equipe multidisciplinar

( ) 5. Ser assegurado com relação à segurança enquanto se encontra no hospital

( ) 6. Conversar sobre sentimentos em relação ao que vem acontecendo

( ) 7. Conversar sobre a possibilidade de falecimento do meu familiar

( ) 8. Ter alguém encarregado sobre minha saúde

( ) 9. Ter alguma outra pessoa comigo quando estiver visitando meu familiar na UTI

( ) 10. Sentir-se a vontade para chorar

( ) 11. Ter a liberdade de ficar sozinho(a) a qualquer hora

( ) 12. Receber a visita de um representante religioso (padre, pastor, rabino)

Outras necessidades (nesse espaço, o $\operatorname{Sr}(a)$ poderá acrescentar outros itens que correspondem a outras necessidades suas, relacionadas à segurança emocional, que não constam na listagem apresentada acima):

D) Necessidade de acesso ao paciente e aos profissionais da unidade: referem-se às necessidades de ter maior acesso ao seu familiar internado na UTI e aos profissionais da instituição (médicos, enfermeiros, fisioterapeutas, nutricionistas, psicólogas, e demais funcionários não relacionados à área médica).

( ) 1. Conversar com o médico todos os dias

( ) 2. Ver o meu familiar freqüentemente

( ) 3. Conversar com a enfermeira responsável todos os dias

( ) 4. Ter o dia e o horário de visita flexíveis, podendo ser modificados em situações especiais

( ) 5. Ter o horário de início da visita respeitado, isto é, iniciar a visita pontualmente no horário previsto

( ) 6. Ter uma pessoa específica no hospital para entrar em contato e ter notícias do meu familiar quando não puder estar presente na visita

( ) 7. Ajudar com relação aos cuidados físico do meu familiar

( ) 8. Poder visitar a qualquer momento

( ) 9. Ter alguém para ajudar com problemas financeiros

( ) 10. Conhecer os cuidados da equipe com relação ao meu familiar

Outras necessidades (nesse espaço, o $\operatorname{Sr}(a)$ poderá acrescentar outros itens que correspondem a outras necessidades suas, relacionadas ao acesso ao seu familiar e aos profissionais da unidade, que não constam na listagem apresentada acima): 